\title{
Analysing knot evidence: associating innate habits with sophisticated tying tasks
}

\author{
Robert Charles Chisnall
}

Kingston, Ontario CANADA, chisnall@kingston.net

\begin{abstract}
:
Most ligature cases feature everyday, innately-tied Overhand Knots, Half Hitches and Half Knots. These knots are the result of habitual behaviour and individual tiers demonstrate consistency, except when certain contextual factors come into play. This survey focussed on comparing the chiralities of basic knots to those of Figure Eight Knots, which occur in case evidence and require similar tying actions. It is important to note that real-world Figure Eights are oriented relative to their working ends and are therefore chiral, whereas topological Figure Eights have no ends and are amphichiral. Data summarizing the tying habits of 184 survey respondents were collected and analysed. The majority of volunteers surveyed tied common Overhand Knots and Figure Eights of equal chirality, consistently or nearly consistently, irrespective of any general learning effect. A minority tied knots of opposite chirality. The knots tied by the remaining respondents varied, and the data suggested a potentially complex pattern which may be related to previous findings. Similar but less pronounced patterns were exhibited in the Half Hitch and Half Knot data. This information could be useful when analysing case evidence and making links to suspect samples, provided cautious attention is paid to context and knot function.
\end{abstract}

Keywords:

forensic; knot; chirality; tying; behaviour

\section{INTRODUCTION, BACKGROUND AND RATIONALE}

Most ligatures found at crime scenes are secured with basic Overhand Knots, Half Hitches, Half Knots and combinations or variations or these structures (Figures 1-3) [1-3]. Knots that indicate some level of tying sophistication and expertise are rarely encountered [4-6]. The occurrence of more sophisticated knots in case evidence - such as Bowlines, Sheet Bends and Figure Eight Knots (Figures 4-6) - is noteworthy because it points to an elevated level of expertise and could narrow the field of potential suspects. However, the prevalence of knots requiring skill beyond the ordinary introduces several unknowns insofar as forensic research is concerned. In particular, details pertaining to exact knot structure and the associated tying behaviour deserve investigation.

Based on years of case work and qualitative observations, one of many questions keeps recurring [1-7]. To what extent do innate tying habits affect more sophisticated tying skills? Any connection in this regard could assist forensic investigators of crime scene knots, allowing them to make groupcharacteristic associations between case exhibits and suspect samples. This question was the motivation for the survey study reported herein.

One of the challenges in undertaking research of this nature is the paucity of individuals in the general population who know how to tie any knots more complex than basic shoelace and packaging or binding knots, which are tied without the benefit of extensive training or skill [1-4]. Therefore, it is difficult to find an adequate number of appropriate survey respondents. Which sophisticated survey knots would garner sufficient data and maximize the number of respondents, and what would those specific knots reveal in relation to innate tying behaviour? In addition to three basic tying tasks - Overhand Knots, Half Hitches and Half Knots - two specialized but relatively mainstream knots were selected for this study: the Figure Eight Knot and the Bowline, the latter of which has been sometimes called the king of knots. These knots were selected for a variety of reasons.

Topologists have long known that the Figure Eight is achiral or amphichiral. It has no mirror image, and any Figure Eight can be superimposed on any other Figure Eight by applying simple mathematical operators from set theory [7]. (Two of these operators are rotations around the $x$ or $y$ axis in order to make one knot match another.) However, it is important to realize that topological knots have no working ends or wends, and they cannot be untied (Figure 7). Real-world knots found in case evidence have wends (Figure 6). A practical Figure Eight is oriented relative to its wend and, hence, it has a characteristic chirality which has been previously ignored by case investigators and researchers [7].

With four crossing points, the Figure Eight is a little more complicated than the simple Overhand Knot, which contains the minimal three crossings required to create a true knot. The Overhand or Trefoil Knot is analogous to the Half Hitch, and both of these everyday knots are typically tied using one wend (Figures 1 and 2). They are similar to basic Half Knots (Figure 3), which are factor knots or components of packaging knots such as the two Granny and two Reef Knots (Figures 8 and 9). These four ubiquitous cardinal knots have been observed in case evidence [1-9].

The Figure Eight is a relatively simple knot to teach novices in order to investigate the degree to which innate habits and learning may come into play. More significantly, the basic manipulations required to create a Figure Eight are somewhat similar to the actions required to tie basic Overhand Knots. Both knots are initiated with an S or Z twist, thereby establishing which enantiomorph will result (Figure 10). There are two basic methods of accomplishing this, particularly with the Figure Eight Knot: create a loop and twist it, or wrap the wend around the standing part. The Overhand Knot is immediately completed by tucking the wend into the Crossing Loop created by the twist, while the tying of the Figure Eight continues with a change of direction and another twist (Figure 11). The resulting structure is an elbow 


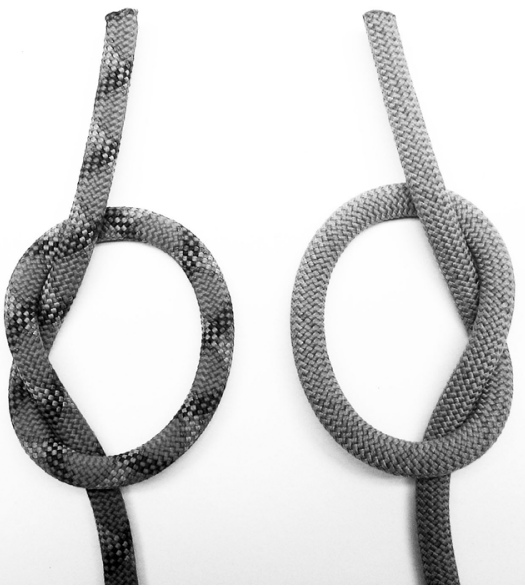

Figure 1. Overhand Knots: left S, right Z.

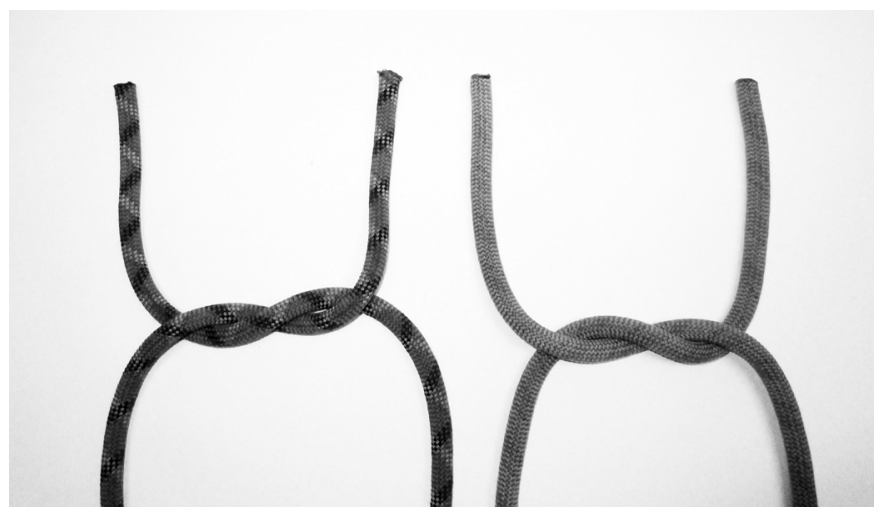

Figure 3. Half Knots: left S, right Z.

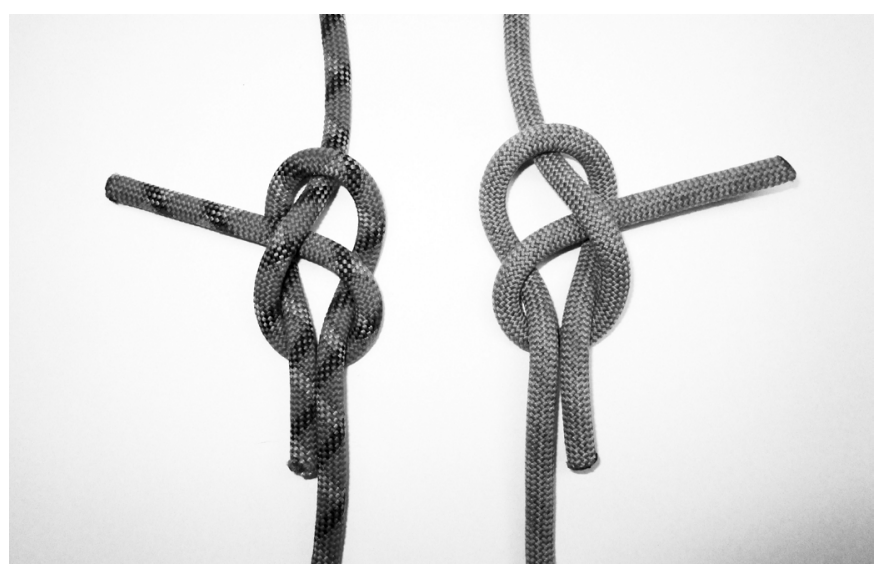

Figure 5. Sheet Bends (direct versions, wends on the same side): left b, right d.

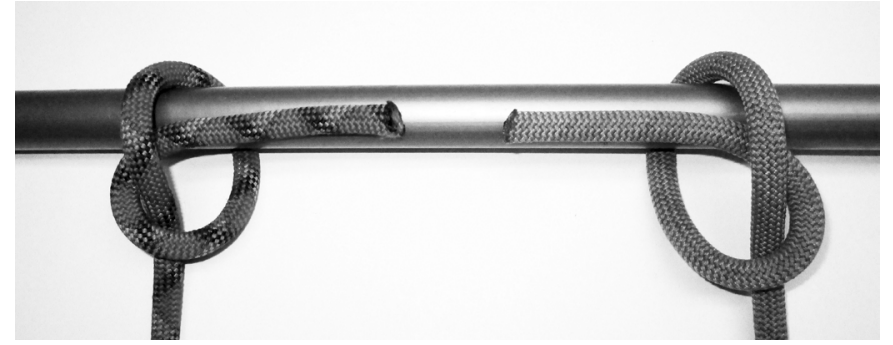

Figure 2. Half Hitches: left S, right Z.

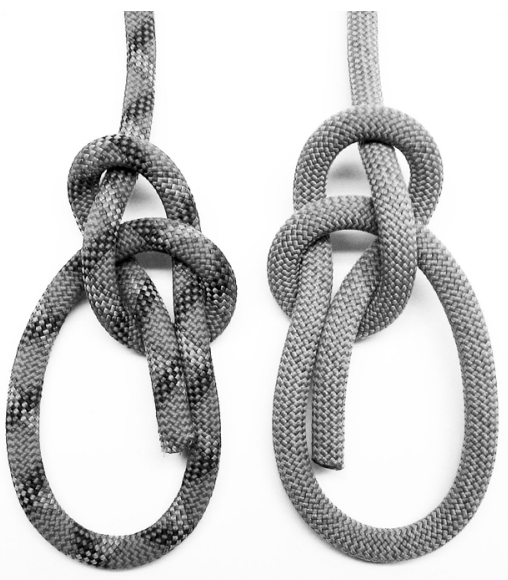

Figure 4. Bowlines: left $b$, right $d$.
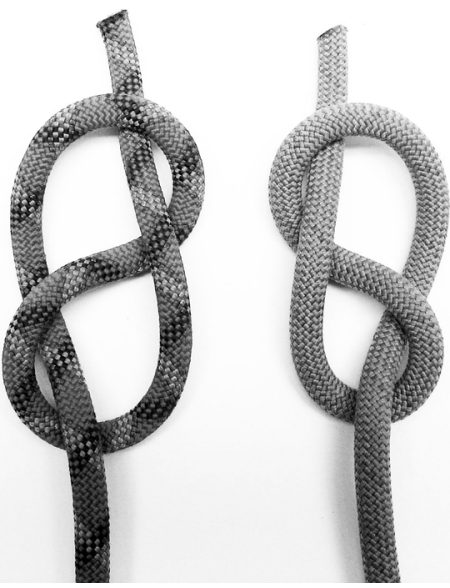

Figure 6. Eight Knots: left S, right Z. 


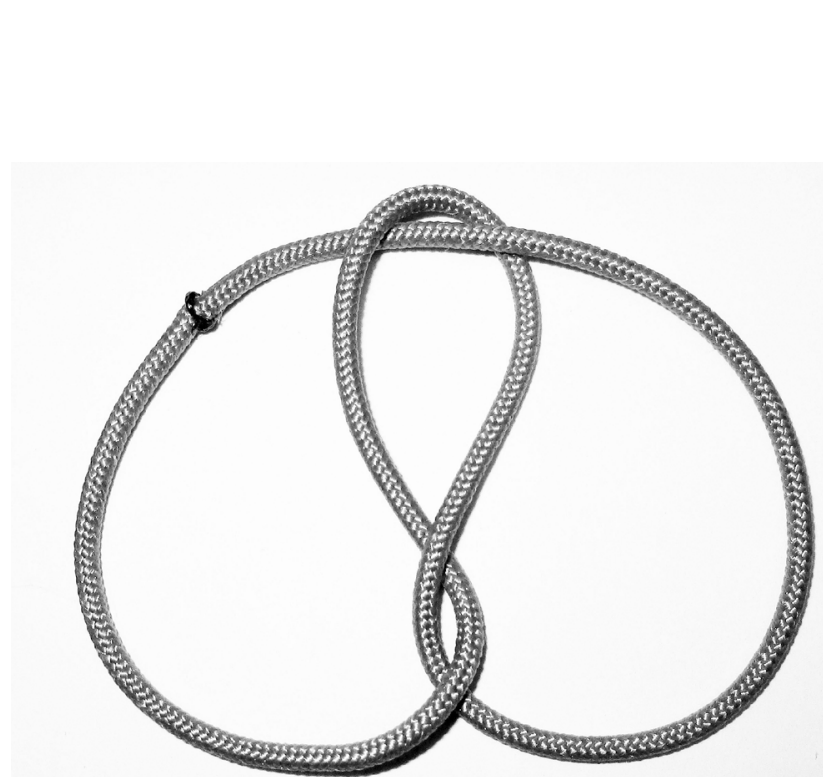

Figure 7. Topological Figure Eight (no wends).

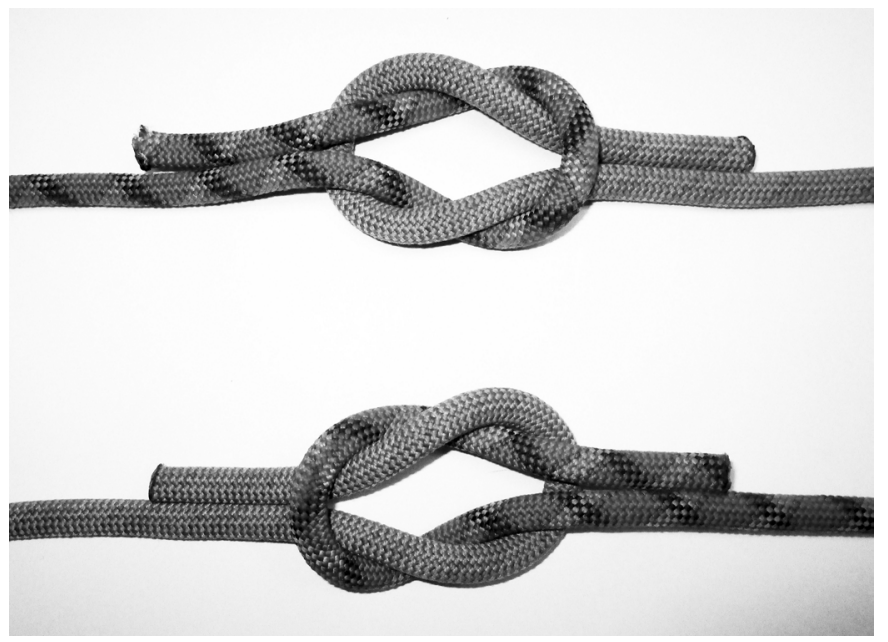

Figure 9. Reef Knots: top S/Z, bottom Z/S.

and the overall configuration is similar to a Crossing Hitch. Then the wend is tucked into the Crossing Loop furthest from the standing part of the cord or rope to complete the Figure Eight Knot (Figure 12). In performing this final step, the tier wraps the wend in the same direction as the initial twist. Consequently, the Figure Eight comprises two tandem Crossing Loops. This tying action may be related to innate behaviour, or it may be an artefact of how the knot is initially learned and practiced.

How is this important to an investigator of crime scene evidence? Figure Eights have been encountered in actual case exhibits, along with the basic knots mentioned at the outset. Figure Eights are used by mountaineers, rescue personnel, sailors and other people with certain recreational and professional specializations $[1,2,8]$. Figure Eights can also occur accidentally $[1,10]$, so clearly identifying knot chirality and function during evidence analyses is vital. Within the context of corroboration and group-characteristic evidence, the question of tying consistency or congruity within a set of case exhibits and between knot evidence and suspect samples could arise.

In contrast, the tying of the Bowline necessitates a different series of actions. In fact it can be created in a number of ways, which will not be

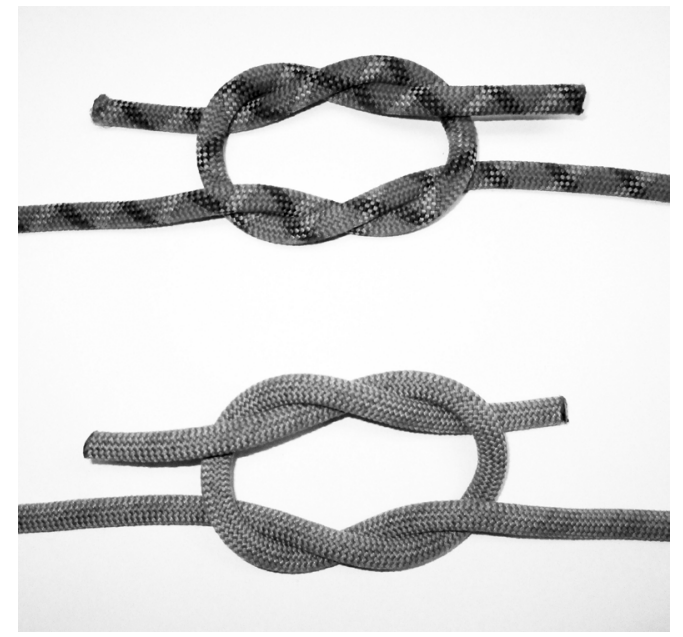

Figure 8. Granny Knots: top S/S, bottom Z/Z.

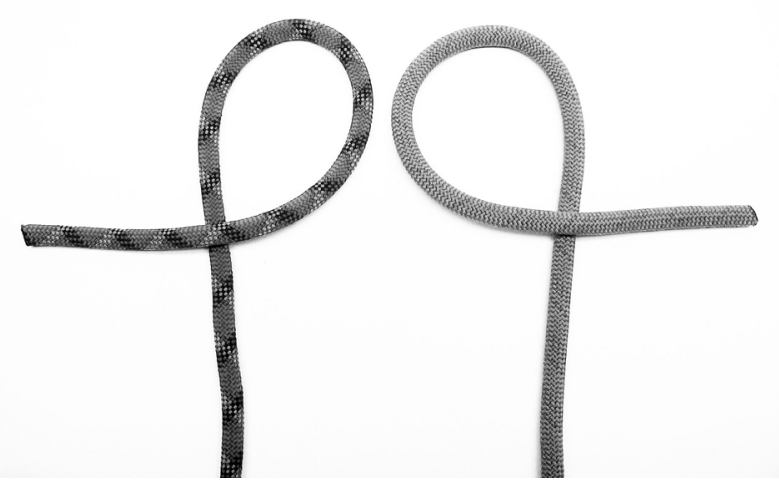

Figure 10. Step 1- Initiating Overhand and Figure Eight Knots with Crossing Loops.

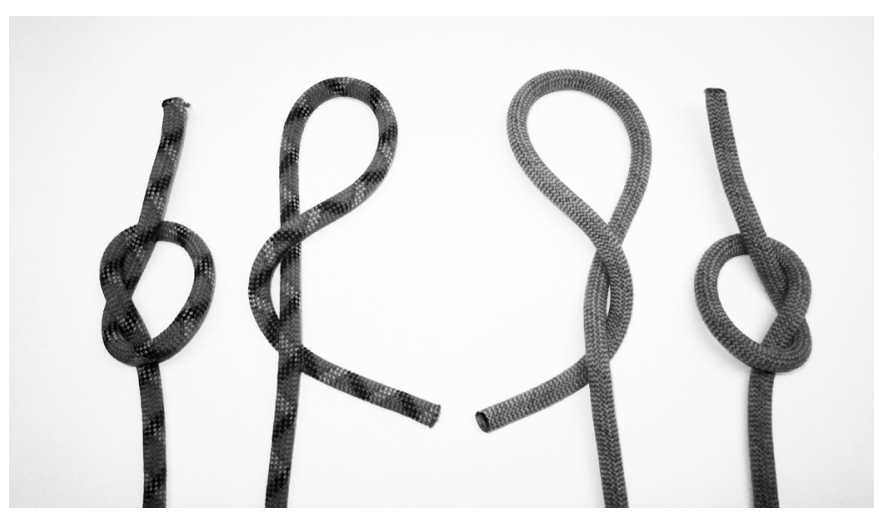

Figure 11. Step 2 - Overhand Knots completed, changing directions to finish Figure Eight Knots with twists or elbows, which look like Crossing Hitches: left S, right Z. 
presented here in any detail: the rabbit-in-the hole method, the musclememory technique, and the Overhand Slip Knot method, to mention just a few tying procedures [11-33]. Insofar as experienced knot tiers are concerned, the Bowline is relatively ubiquitous, figuring in a variety of recreational and professional specialities such as sailing, industrial rigging, climbing and rescue work [12, 18, 29, 30, 32, 33]. In theory there should be a greater chance of finding more survey volunteers who can actually tie this knot, relative to tiers of more esoteric formations. As with the Figure Eight Knot, similar questions emerge. Do innate habits have any relationship to the actions required to tie a Bowline and to its completed structure? Like Overhand Knots and Half Knots, Bowlines are also chiral, appearing as either b or d enantiomorphs (Figure 4), which can potentially distort or capsize into S or Z Slip Knots or Running Slip Loops, respectively, as shown in Figure 13. Could the form of the Bowline tied be the result of training alone or do innate tendencies influence the result? Certainly it can be observed that a right-handed person will produce a d Bowline when performing the onehanded or seaman's method, and a left-handed person will tie the opposite form. However, this tendency is not so obvious or consistent with other tying methods.

\section{SOME PRELIMINARY OBSERVATIONS}

It has been reported that comparatively sophistciated knots, like the Figure Eight Knot and the Bowline, occurred in less than $5 \%$ of cases examined [1, $2,6]$. Out of 105 cases analysed up to the time of the published data [6], six featured one or more Figure Eight Knots or Figure Eight Slip Loops (not illustrated). However, the Eight Knots observed in evidence from three of those cases may have been accidental, and the chirality of most of the Figure Eights was not recorded. The Bowline and the Sheet Bend (Figures 4 and 5), the latter of which is structurally analogous to the Bowline, were observed in three cases. A single d Bowline appeared in one case. A second case presented a single b Bowline along with mainly S Overhand Knots and Half Hitches. A single S-configured Figure Eight was preserved in a third case, amongst other simpler scene knots including pairs of $Z$ and $S$ Half Hitches. The fourth and most interesting case involved the identification of numerous Bowlines and Figure Eights throughout a large collection of suspect samples and scene exhibits. At least $11 \mathrm{~S}$ Figure Eights were observed. Not a single $Z$ Figure Eight was documented, and it was noted that out of more than one

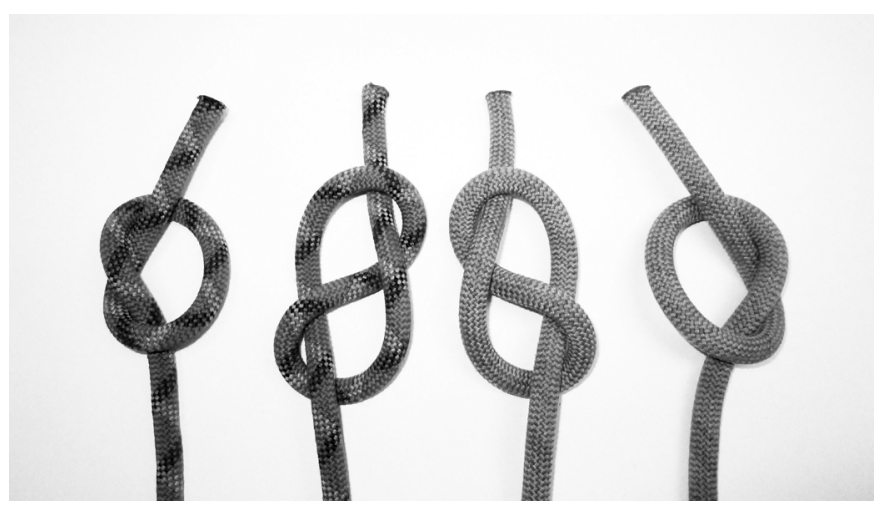

Figure 12. Step 3 - Overhand and Figure Eight Knots completed and compared: left S, right Z. hundred simple Half Knots, Overhand Knots and Half Hitches, most were $\mathrm{S}$ enantiomorphs. Additionally, amongst a number of other sophisticated knots associated with sailing and knotting expertise, at least $20 \mathrm{~d}$ Bowlines and not a single $b$ Bowline were encountered in the exhibits.

The latter observations verify previous assertions that tying habits are consistent and repeatable [1-7, 10]. More to the point here, such anecdotal details hint that there may be a link between the chirality of innately tied Overhand Knots, Half Knots and Half Hitches, and that of more sophisticated knots requiring some expertise, like Figure Eights and perhaps Bowlines. The number of knot cases analysed since that initial meta-analysis [6] has grown, and the same incidence of sophisticated knots and similar chirality patterns have been observed.

At the outset of the research reported herein, it was speculated that there may be a prevalent learning effect based on instructor preferences and perhaps on how leading knot publications present chiral knots. An evaluation of authoritative texts and lesser-known publications offered moderate insight [1, 11-33], as shown in Table 1. This was a rough sample of the manifold books on knots currently available or out of print. The tabulated references shared basic commonalities, but they also presented esoteric differences in terms of general nomenclature, certain knot names and methods of tying [7]. Most featured at least a few unique details.

Twenty-four references from different decades and countries were included in this literature survey, some of which are well known to knot afficionados while others are obscure. It was assumed that the knots depicted in each book were a fair representation of the author's tying habits. A minority of the authors sampled were aware of the various chiral versions of the knots documented. Instances of Overhand Hand Knots, Figure Eight Knots, Bowlines and Sheet Bends were noted, and enantiomer prevalence has been listed in Table 2 . The number of chiral versions of knots presented in each book is equal to or greater than the number tabulated, depending on how structural variants are interpreted. The numbers listed constitute a reasonable overview of the knots represented by each author. The drawings or photographs illustrating each book were not necessarily rendered by those authors. As Tables 1 and 2 summarize, the $d$ Bowline was depicted most frequently. Although there was a preponderance of S Figure Eight Knots and related formations, chirality was not always consistent within texts illustrating multiple instances of the Figure Eight. Similarly, there was no regularity or pattern in the depiction of basic Overhand Knots and Bowlines.

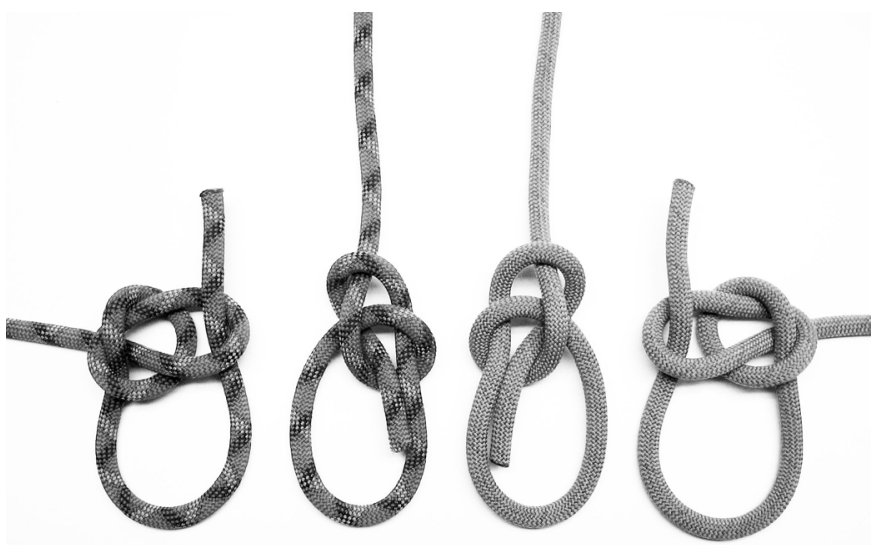

Figure 13. Bowline capsizement. Inside: right b Bowline, left d Bowline. Outside, Running Slip Loops or Knot: left S, right Z. 
Table 1. A survey of knot chirality in 24 knot references [1, 11-33].

\begin{tabular}{|c|c|c|c|c|}
\hline Print Source & Overhand Knots & Figure Eights & Bowlines & Sheet Bends \\
\hline Asher (1989) & $14 Z, 4 S$ & $2 S$ & $7 d$ & \\
\hline Ashley (1944) ${ }^{1,3}$ & $18 \mathrm{Z}, 36 \mathrm{~S}$ & $4 Z, 10 S$ & $4 \mathrm{~d}, 1 \mathrm{~b}$ & $19 \mathrm{~d}, 9 \mathrm{~b}$ \\
\hline Berthier (1974) ${ }^{1}$ & $3 \mathrm{Z}, 5 \mathrm{~S}$ & $1 \mathrm{~S}$ & $6 d$ & \\
\hline Bigon \& Regazzoni (1981) ${ }^{2}$ & $6 \mathrm{Z}, 6 \mathrm{~S}$ & $5 Z$ & $11 \mathrm{~d}$ & $1 \mathrm{~d}, 4 \mathrm{~b}$ \\
\hline Blandford (1965) & $3 \mathrm{Z}, 10 \mathrm{~S}$ & $1 \mathrm{Z}, 4 \mathrm{~S}$ & $1 \mathrm{~d}$ & $1 \mathrm{~d}, 2 \mathrm{~b}$ \\
\hline Bourgault (1985) & $8 \mathrm{Z}, 5 \mathrm{~S}$ & $6 \mathrm{Z}, 1 \mathrm{~s}$ & $9 d$ & $5 \mathrm{~b}$ \\
\hline Brown and Hunter (1977) ${ }^{2}$ & $2 \mathrm{Z}, 6 \mathrm{~S}$ & $1 \mathrm{Z}, 1 \mathrm{~S}$ & $4 d$ & $2 b$ \\
\hline Budworth (1983) & $4 Z, 10 S$ & $1 \mathrm{Z}, 2 \mathrm{~S}$ & $2 d$ & $1 \mathrm{~d}$ \\
\hline Budworth (1999) & $12 \mathrm{Z}, 3 \mathrm{~S}$ & $2 \mathrm{Z}, 4 \mathrm{~S}$ & $9 d$ & $2 b$ \\
\hline Burgess (1979) & $11 \mathrm{Z}, 2 \mathrm{~S}$ & $2 Z$ & $3 b$ & $5 b$ \\
\hline Chisnall (2000) ${ }^{1,5}$ & $5 \mathrm{Z}, 8 \mathrm{~S}$ & $1 \mathrm{Z}, 4 \mathrm{~S}$ & $3 \mathrm{~b}$ & $1 \mathrm{~d}$ \\
\hline Day (1953) & $4 \mathrm{Z}, 3 \mathrm{~S}$ & $1 \mathrm{~S}$ & $5 d$ & $1 \mathrm{~d}, 2 \mathrm{~b}$ \\
\hline Fletcher (1976) & $2 \mathrm{Z}, 9 \mathrm{~S}$ & $6 \mathrm{~S}$ & $1 \mathrm{~d}, 1 \mathrm{~b}$ & $1 \mathrm{~d}, 1 \mathrm{~b}$ \\
\hline Fry (1977) & $1 \mathrm{Z}, 2 \mathrm{~S}$ & $1 \mathrm{~S}$ & $2 d$ & $2 \mathrm{~b}$ \\
\hline Gibson (1979) & $2 \mathrm{Z}, 3 \mathrm{~S}$ & $2 S$ & $1 \mathrm{~d}$ & $1 \mathrm{~b}$ \\
\hline Graumont \& Hensel (1939) ${ }^{2,3}$ & $7 \mathrm{Z}, 4 \mathrm{~S}$ & $1 \mathrm{Z}, 3 \mathrm{~S}$ & $14 \mathrm{~d}, 5 \mathrm{~b}$ & $4 \mathrm{~d}, 4 \mathrm{~b}$ \\
\hline Jacobson $(2008)^{5}$ & $4 \mathrm{Z}, 2 \mathrm{~S}$ & $1 \mathrm{Z}, 1 \mathrm{~S}$ & $1 \mathrm{~d}, 1 \mathrm{~b}$ & $2 \mathrm{~d}, 2 \mathrm{~b}$ \\
\hline “Knots” (1998) & $4 \mathrm{Z}, 5 \mathrm{~S}$ & $2 \mathrm{Z}, 1 \mathrm{~S}$ & $6 d$ & $1 \mathrm{~b}$ \\
\hline Luebben (1993) & $3 \mathrm{Z}, 2 \mathrm{~S}$ & $4 \mathrm{Z}, 1 \mathrm{~s}$ & $1 \mathrm{~b}$ & \\
\hline Pawson (2001) & $7 \mathrm{Z}, 8 \mathrm{~S}$ & $5 \mathrm{~S}$ & $2 d$ & $2 \mathrm{~b}$ \\
\hline Raleigh (1998) ${ }^{4}$ & $1 \mathrm{Z}, 7 \mathrm{~S}$ & $4 Z, 13 \mathrm{~S}$ & $3 d$ & \\
\hline Shaw (2003) & $5 \mathrm{Z}, 5 \mathrm{~S}$ & $1 \mathrm{Z}, 1 \mathrm{~S}$ & $7 d$ & $3 b$ \\
\hline Warner (1992) ${ }^{1,5}$ & $17 \mathrm{Z}, 5 \mathrm{~S}$ & $1 \mathrm{Z}, 9 \mathrm{~S}$ & $14 \mathrm{~d}, 3 \mathrm{~b}$ & $4 \mathrm{~d}, 4 \mathrm{~b}$ \\
\hline Wheelock (1960) ${ }^{4}$ & $11 \mathrm{Z}, 3 \mathrm{~S}$ & $2 \mathrm{Z}, 1 \mathrm{~S}$ & $6 b$ & $2 b$ \\
\hline
\end{tabular}

${ }^{1}$ The authors are known to have illustrated their books.

${ }^{2}$ These books are the work of two authors.

${ }^{3}$ Just a sample of the knots presented in these major references are tabulated.

${ }^{4}$ The books listed are specialized climbing monographs featuring the Figure Eight Knot and Bowline.

${ }^{5}$ To a certain extent, the chirality of knots was addressed in these references.

Table 2. A summary of knot chirality data from reference survey.

\begin{tabular}{|c|c|c|c|c|}
\hline Knots & All or MostlyS & All or Mostly Z & Equal Counts & Blank Cells \\
\hline Overhand Knots & 12 & 10 & 2 & 0 \\
\hline Figure Eight Knots & 15 & 6 & 3 & 0 \\
\hline Knots & All or Mostly b & All or Mostly d & Equal Counts & Blank Cells \\
\hline Bowlines & 4 & 18 & 2 & 0 \\
\hline Sheet Bends & 13 & 3 & 4 & 4 \\
\hline Knot Comparisons & $\begin{array}{c}\text { Consistent or Mainly Con- } \\
\text { sistent S }\end{array}$ & $\begin{array}{c}\text { Consistent or Mainly Con- } \\
\text { sistent Z }\end{array}$ & $\begin{array}{c}\text { Opposite or Mainly } \\
\text { Opposite }\end{array}$ & $\begin{array}{c}\text { Equal Counts } \\
\text { Either Cell }\end{array}$ \\
\hline Overhand \& Eight Knots & 10 & 4 & 6 & 4 \\
\hline Overhands \& b Bowlines & 1 & 3 & 0 & 4 in Total: \\
\hline \multirow[t]{2}{*}{ Overhands \& d Bowlines } & 10 & 6 & 0 & $2 S=Z$ \\
\hline & & & & $2 b=d$ \\
\hline Knot Comparisons & Consistent or Mainly b & Consistent or Mainly & All or Mainly Opposite & $\begin{array}{c}\text { Equal Counts, } \\
\text { Either Knot } \\
\text { (Blank Cells) }\end{array}$ \\
\hline Bowlines \& Sheet Bends & 2 & 2 & 12 & $4(4)$ \\
\hline
\end{tabular}


These initial observations do not highlight any obvious relationship between innate tying and sophisticated skills in terms of knot chirality and specific structural details.

\section{METHOD}

Over a period of three years, volunteer participants in six separate groups provided useful survey data - three smaller survey groups, whose data were combined into one larger group, and three larger groups. The rationale for these divisions goes beyond simple participant availability. Specific and relatively variable knotting experience amongst sets of respondents was most important. Adequate working space, appropriate context and ample available time were also factors. The survey sites were spacious classrooms or meeting areas with tables, chairs and good lighting.

Three smaller sample groups of climbing instructors, climbing gym members, and climbing club members and one large group of climbers was surveyed because they use Figure Eight Knots to tie the safety rope to their harnesses on a regular basis. It was presumed there would be a higher chance members of such groups would know the Bowline as well, which they were asked to tie if they could.

The other two larger groups comprised university students who did not necessarily have any prior specialized knot-tying experience. Projected images and demonstrations were utilized to teach these groups the $Z$ version of the Figure Eight as part of the survey procedure to test if there was a general training effect. Inexperienced knot tiers were asked to tie a series of three Figure Eights over a period of two or three hours to assess retention and consistency. These unskilled respondents were not asked to tie Bowlines.

Participants recorded the structure of the knots they tied on their survey forms. Print materials and projected images were made available to assist in knot identification. The survey facilitator circulated amongst survey participants to verify knot identifications, or to correctly identify knots when there was uncertainty. In conjunction with the Figure Eight and Bowline tying tasks, all respondents were directed to tie some basic knots at least three times: Overhand Knots and Half Hitches, which typically require the manipulation of one wend, and parcelling knots comprising component Half Knots, which necessitate the handling of two wends simultaneously.

A limited variety of tying materials was made available to volunteers. Cordage ranged in length from $150 \mathrm{~cm}$. to $1,000 \mathrm{~cm}$., and from four to eight millimetres in diameter. All cords were of similar pliability, which is also known as knotability or working capacity in some fields [34-36]. Survey cords were either braided nylon or polypropylene with melted ends. To avoid any chance that participant tying behaviour might be affected by survey material asymmetries, no hawser-laid ropes were employed in the survey. (Hawser-laid rope is typically $Z$ lay and therefore chiral.)

\section{RESULTS}

Not all survey volunteers completed every task adequately. Only data from those individuals who successfully accomplished the tying tasks under comparison were tabulated. A total of 184 respondents submitted usable surveys, and those participants consisted of 67 males and 117 females ranging from 18 to 46 years of age. Most respondents were Canadian. However, a minority of survey volunteers hailed from Taiwan, France, Switzerland, Oman,
Australia and Lebanon. Only six sinistral and one ambidextral respondent participated in this study, which is below the expected number for a sample group of this size - one in 25, rather than the average one in 10 to 14 [37-44]. This was an insufficient number of left-handed respondents for comparison purposes, so the data were not analysed in consideration of tier handedness. (A previously published analysis of basic knot-tying habits indicated a subtle, complex and nonbinary relationship between the chiralities of fundamental, innately-tied knots and tier handedness [3].)

\section{BOWLINE DATA}

With regard to Bowline tying behaviour, very little information was acquired and this outcome was immediately anticipated after the first group was surveyed. The most likely individuals who might have been able to tie Bowlines were the climbers. However, most modern climbers no longer use the Bowline as a harness tie-in technique for security reasons.

The Bowline is a post-bight knot and it can invert into a Slip Knot or, more accurately, a Running Slip Knot (Figure 13) which subsequently can come untied tragic consequences [45, 46]. There have been numerous cases of Bowline failures resulting in near-misses, severe accidents and even fatalities. On the other hand, the Figure Eight Follow-Through (not illustrated) is a pre-bight knot and cannot invert or come loose if initially tied and completed correctly $[45,46]$. The question of knot security is beyond the scope of this paper and it is more applicable to civil cases.

Out of 68 volunteer climbers who might have possessed the skill to tie a Bowline, only 19 claimed they had that ability. However, twelve of those respondents had to be coached. Toward that end, the survey facilitator demonstrated the $d$ enantiomorph and the participants subsequently produced d Bowlines with some hesitation. A general learning outcome was immediately apparent and no link to innate tying habits could be made owing to the lack of unbiased data. The remaining seven volunteers could actually tie Bowlines without assistance. Four tied the $d$ enantiomorph, two produced the $b$ Bowline, and one individual tied both versions. The chirality of the small number of basic knots and Figure Eight Knots tied by these seven volunteers was mixed and there was no apparent pattern linking Bowline chirality to innate tying habits. Further, it was realized during the administration of this survey that the tying actions involved in creating Bowlines is quite different from those required to produce Overhand Knots and Figure Eight Knots, as mentioned earlier and noted by other authorities [11-33].

\section{FIGURE EIGHT VERSUS OVERHAND KNOT DATA}

The data acquired from the tying of basic Overhand Knots and Figure Eight Knots were substantial and more revealing. Similar cohort ratios were replicated in each of the four sample groups. A z-test comparing the six pairings of the four major sample groups showed no significant difference. The ratio of consistent to contrary tiers was equivalent - consistent tiers being those whose Overhand and Figure Eight Knots were of equal chiraity, while contrary tiers produced Overhand Knots that were opposite in chirality to their Figure Eight Knots.

A chi-squared test using a five-by-five contingency table of raw Overhand Knot data, as shown in the top half of Table 3, yields a chi-squared value of 52.8469 with a p-value less than 0.00001 . This result is significant, 
.

and similar outcomes can be generated in the same manner for the Half Hitch and Half Knot data (Tables 4 and 5). However, this may be misleading because prior research $[3,5,6]$ indicates that $S, Z$ and mixed Overhand Knot, Half Hitch and Half Knot tiers are not equally distributed in the general population. Based on this previous observation, there is an expectation that the majority of tiers will be $S$ or mainly $S$ tiers, the $Z$ or mainly $Z$ tiers will be a significant minority, and a very small minority will be mixed tiers. The survey data presented herein agree with these expectations.

As the upper half of Table 3 summarizes, 79 participants out of 184, or $43 \%$, consistently tied Figure Eight Knots of the same chirality as their Overhand Knots, without deviation, indicating a behavioural link. This association can be made more apparent if the number of individuals who tied the majority of their Overhand Knots and Figure Eights as either S or Z enantiomorphs is included in the total, as shown in the lower half of Table 3. A total of 105 respondents, or 57\%, tied Figure Eights and Overhand Knots mainly of the same chirality. Not surprisingly, since most tiers in the general population produce S Overhand Knots and other basic knots [1-6], a majority of the survey Figure Eights were $S$ enantiomorphs irrespective of the fact that the Z Figure Eight Knot was demonstrated to both large, inexperienced survey groups. There was no discernible learning effect.

Only $33 \%$ of the volunteers surveyed were contrary tiers, producing Figure Eights which were mainly opposite in chirality to most of their Overhand Knots. Partially mixed tiers, those who tied either an even number of S and Z Figure Eights or Overhand Knots but not both, amounted to $8 \%$ of the total. Truly mixed tiers - those without any apparent chirality preferences during both tasks, made up less than $2 \%$ of the sample. However, the individual patterns exhibited within the latter three groups were more complex than just a random mixture of enantiomers, and this observation invites further investigation. There may have been a recency and primacy effect based on tying order, as previously observed in a survey of case evidence [6].

The statistical significance of these data can be analysed in several ways based on expected values using different assumptions. Previous investigations indicate that chirality preference is consistent and repeatable for basic knots like the Half Hitch, Overhand and Half Knot, unless specific reversal factors come into play [1-7]. Therefore, irrespective of Overhand Knot chirality preference, if it is assumed that there should be an even distribution of Figure Eight tiers among the three major cohorts - S and Sz tiers, sz tiers, and $s Z$ and $Z$ tiers, as summarized at the bottom of Table 3 - the chi-squared value is 72.711 , and the $p$-value is less than 0.001 , which is significant. The Fisher exact test for small populations yields similar results for this and subsequent analyses. Therefore, the pattern of tiers spread between the three cohorts is not random. The chi-squared test can be further applied to each row of data, with the same outcome for the strongly $S$ and $Z$ Overhand Knot tiers. The central, mixed row contains a small number of tiers and the pattern is not significant.

The second method of evaluating the data is to assume that the number of Figure Eight tiers should be spread evenly amongst consistent, contrary and mixed tiers. A chi-squared value of 60.134 results, with a $p$-value less than 0.001 . The pattern is significant, but the chirality association between Overhand and Figure Eight Knots is not absolute. This result should be regarded as a potential tool for evidence corroboration from a groupcharacteristic standpoint. Knot-tying habits cannot be used as individual characteristics like finger prints and DNA [1-3].

\section{FIGURE EIGHT VERSUS HALF HITCH DATA}

Survey volunteers also were asked to tie multiple Half Hitches around a fixed object using one wend of their survey cord. The number of Half Hitch tiers can be analysed according to the chiralities of the Figure Eights they

Table 3. A comparison of Figure Eight Knot and Overhand Knot chiralities according to the number of tiers. Top: raw data. Bottom: similar cohorts grouped together.

\begin{tabular}{|c|c|c|c|c|c|c|}
\hline \multirow{2}{*}{$\begin{array}{l}\text { Chirality of Overhand Knots tied by Figure Eight } \\
\text { Knot tiers }\end{array}$} & \multicolumn{5}{|c|}{ Number of Figure Eight tiers according to knot chirality } & \multirow[b]{2}{*}{ Totals } \\
\hline & $S$ & $\mathrm{Sz}$ & SZ & $s Z$ & Z & \\
\hline S & 52 & 8 & 3 & 11 & 16 & 90 \\
\hline Sz & 4 & 3 & 0 & 2 & 7 & 16 \\
\hline sz & 5 & 1 & 3 & 1 & 3 & 13 \\
\hline$s Z$ & 6 & 5 & 1 & 5 & 4 & 21 \\
\hline Z & 12 & 2 & 1 & 2 & 27 & 44 \\
\hline Totals & 79 & 19 & 8 & 21 & 57 & 184 \\
\hline
\end{tabular}

\begin{tabular}{|c|c|c|c|c|}
\hline \multirow{2}{*}{$\begin{array}{l}\text { Chirality of Overhand Knots tied by Figure Eight } \\
\text { Knot tiers }\end{array}$} & \multicolumn{3}{|c|}{ Number of Figure Eight tiers according to knot chirality } & \multirow[b]{2}{*}{ Totals } \\
\hline & $\mathrm{S}, \mathrm{Sz}$ & SZ & $s Z, Z$ & \\
\hline $\mathrm{S}, \mathrm{Sz}$ & 67 & 3 & 36 & 106 \\
\hline sz & 6 & 3 & 4 & 13 \\
\hline$s Z, Z$ & 25 & 2 & 38 & 65 \\
\hline Totals & 98 & 8 & 78 & 184 \\
\hline
\end{tabular}

S = Tied all S; Sz = Tied mostly S; sZ = Tied S and Z equally; $\mathbf{Z}$ = Tied mostly Z; Z = Tied all Z.

Consistent tiers produced Overhand Knots and Figure Eights that were all or nearly all $S$ or $Z$.

Contrary tiers produced Overhand Knots that were all or nearly all opposite to their Figure Eights.

Mixed tiers produced equal numbers of $S$ and $Z$ enantiomers when tying Overhand Knots or Figure Eights or both. 
produced. Those results are summarized in the upper half of Table 4 in the same way the data are presented for Overhand Knot tiers in Table 3. However, fewer tiers completed the Half Hitch task and it should be pointed out that, although one wend is used to tie a simple hitch with three crossings, which is analogous to the Overhand or Trefoil Knot, the task is different. An Overhand Knot can be tied in one wend independent of its environment, like a Figure Eight, whereas Half Hitches are tied around oriented secondary objects.

The results present ratios somewhat akin to those apparent in the Overhand Knot versus Figure Eight data. Even though the z-test indicates that the proportions of consistent and contrary Figure Eight tiers according to the Overhand Knot task and the Half Hitch task are not significantly different, the ratios for the mixed cohorts are dissimilar. There were more mixed tiers during the Half Hitch task compared to the Overhand Knot task, and this accumulative change came from the few individuals who shifted from the consistent and contrary categories. Even though the patterns of Figure Eight chiralities are similar, the tying of Overhand Knots and Half Hitches are slightly different tasks, thus altering the behaviour of a small number of tiers.

If the chi-squared test for of goodness fit of estimated values is applied to the data in the lower half of Table 4, the grouped cohorts, the result is significant. The chi-squared test yields a value of 48.647 with a $p$-value less that 0.001 . If the three cohorts for the Overhand Knot tiers are assessed individually according to each row of data, the outcomes are also significant.

Assuming the distribution of Figure Eight tiers amongst the collectively consistent, contrary and mixed cohorts should be even, those estimated values produce a chi-squared value of 7.069 with a $p$-value of 0.029 , which is above the 0.01 significance cutoff limit but below the 0.05 limit. Hence, the pattern of Figure Eight Knot chiralities is not as pronounced with the Half Hitch data as it is with the Overhand Knot data. Forty-five percent of Half Hitch tiers were consistent or nearly consistent Figure Eight tiers, 33\% were contrary or mainly contrary Figure Eight tiers, and $25 \%$ were mixed in one or both tasks.

\section{FIGURE EIGHT VERSUS HALF KNOT DATA}

Another basic task surveyed was the tying of Half Knots, which requires the use of two wends rather than one. This is the kind of action necessary to tie binding knots such as Reef and Granny Knots, each comprising two Half Knots. The data are presented in Table 5 according to the prevalence of individual Half Knots. Table 6 summarizes the same data according to the cardinal knots (Reef and Grannies) or the pairs of Half Knots each respondent tied [7].

The same two techniques for creating estimated values, which were employed in analysing Overhand Knot and Half Hitch tiers, can be applied to the Half Knot data. Assuming the number of Half Knot tiers amongst each of the three Figure Eight Knot chirality cohorts should be equal, the chisquared test produces a value of 45.783 with a p-value of 0.001 . The data pattern is unlikely random. Varying levels of significance can be derived by assessing each row of Half Knot tier data separately. However, when the second assumption is used to create estimated values that are equal amongst consistent, contrary and mixed Figure Eight tiers, the chi-squared value is 5.763 at a p-value of 0.056 , which is above even the 0.05 cutoff limit for significance. The pattern is less significant than that exhibited by the Overhand Knot data.

The Half Knot tiers are distributed in a similar way to the Half Hitch tiers. In this case only $42 \%$ were consistent Figure Eight tiers, 33\% were contrary tiers and $25 \%$ produced mixed chiralities. The $z$-test indicates that these ratios are analogous to Half Hitch and Half Knot tier data but, again, the Half Knot ratios are somewhat different from those exhibited in the Overhand Knot data.

The raw cardinal knot data presented at the top of Table 6 can be

Table 4. A comparison of Figure Eight Knot and Half Hitch chirality according to number of tiers. Top: raw data. Bottom: similar cohorts combined.

\begin{tabular}{|c|c|c|c|c|c|c|}
\hline \multirow{2}{*}{$\begin{array}{l}\text { Chirality of Half Hitches tied } \\
\text { by Figure Eight Knot tiers }\end{array}$} & \multicolumn{5}{|c|}{ Number of Figure Eight tiers according to chirality } & \multirow[b]{2}{*}{ Totals } \\
\hline & $S$ & $\mathrm{Sz}$ & SZ & sz & Z & \\
\hline $\mathrm{s}$ & 21 & 3 & 1 & 4 & 6 & 35 \\
\hline $\mathrm{Sz}$ & 5 & 2 & 0 & 1 & 5 & 13 \\
\hline sz & 10 & 2 & 2 & 4 & 13 & 31 \\
\hline$s Z$ & 6 & 2 & 0 & 5 & 9 & 22 \\
\hline z & 10 & 1 & 2 & 1 & 10 & 24 \\
\hline Totals & 52 & 10 & 5 & 15 & 43 & 125 \\
\hline
\end{tabular}

\begin{tabular}{cccc}
\hline \multirow{2}{*}{$\begin{array}{c}\text { Chirality of Half Hitches tied by Figure } \\
\text { Eight Knot tiers }\end{array}$} & \multicolumn{3}{c}{ Number of Figure Eight tiers according to chirality } \\
\cline { 2 - 4 } & S, Sz & Totals & sZ, Z \\
\hline S, Sz & 31 & 1 & 16 \\
SZ & 12 & 2 & 17 \\
Z, sZ & 19 & 2 & 25 \\
Totals & $\mathbf{6 2}$ & $\mathbf{5}$ & $\mathbf{5 8}$ \\
\hline
\end{tabular}

$\mathrm{S}=$ Tied all S; Sz = Tied mostly S; sz = Tied S and Z equally; $\mathrm{SZ}=$ Tied mostly $\mathrm{Z} ; \mathrm{Z}=$ Tied all $\mathrm{Z}$.

Consistent tiers produced Half Hitches and Figure Eights that were all or nearly all $S$ or $Z$.

Contrary tiers produced Half Hitches that were all or nearly all opposite to their Figure Eights.

Mixed tiers produced equal numbers of $\mathrm{S}$ and $\mathrm{Z}$ enantiomers when tying Half Hitches or Figure Eights or both. 


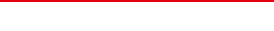

assessed using a contingency table chi-squared test. The result is not significant, with a chi-squared value of 18.7464 and a $p$-value of 0.282035 . This may be meaningless because previous research [3] indicates that there is complex hierarchy of cardinal knot tying preferences relative to other basic knotting habits and the tying of shoelaces, which entails the use of bowed versions of the four cardinal knots. Using the assumption that tier numbers should be equal amongst the three Figure Eight chirality cohorts, the chi-squared test for goodness of fit yields significant values for the $S / S$, $Z / Z, Z / S$ and mixed rows of data, but with a higher $p$-value for $Z / S$ tiers, and a value for the $S / Z$ row of data that is not significant. This suggests that the incidence of Reef Knots adds another layer of complexity to the data, and that the relationship between Figure Eight Knot and cardinal knot chiralities is not as straightforward as it is with the Overhand Knot data.

Table 5. A comparison of Figure Eight Knot and Half Knot chirality according to the number of tiers. Top: raw data. Bottom: similar cohorts combined.

\begin{tabular}{|c|c|c|c|c|c|c|}
\hline \multirow{2}{*}{$\begin{array}{l}\text { Chirality of Half Knots tied } \\
\text { by Figure Eight Knot tiers }\end{array}$} & \multicolumn{5}{|c|}{ Number of Figure Eight tiers according to chirality } & \multirow[b]{2}{*}{ Totals } \\
\hline & $S$ & $\mathrm{Sz}$ & sz & $s Z$ & Z & \\
\hline $\mathrm{S}$ & 29 & 6 & 4 & 2 & 18 & 59 \\
\hline $\mathrm{Sz}$ & 5 & 3 & 0 & 1 & 3 & 12 \\
\hline sz & 8 & 1 & 4 & 2 & 14 & 29 \\
\hline sZ & 9 & 0 & 0 & 1 & 1 & 11 \\
\hline Z & 10 & 2 & 2 & 1 & 12 & 27 \\
\hline Totals & 61 & 12 & 10 & 7 & 48 & 138 \\
\hline
\end{tabular}

\begin{tabular}{cccc}
\hline $\begin{array}{c}\text { Chirality of Half Knots tied by Figure } \\
\text { Eight Knot tiers }\end{array}$ & \multicolumn{3}{c}{ Number of Figure Eight tiers according to chirality } \\
\cline { 2 - 4 } & S, SZ & SZ & TZ, Z \\
\hline S, Sz & 43 & 4 & 24 \\
SZ & 9 & 4 & 16 \\
Z, sZ & 21 & 2 & 15 \\
Totals & 73 & 10 & 53 \\
\hline
\end{tabular}

$\mathrm{S}=$ Tied all $\mathrm{S} ; \mathrm{SZ}=$ Tied mostly $\mathrm{S} ; \mathrm{sZ}=$ Tied $\mathrm{S}$ and $\mathrm{Z}$ equally; $\mathrm{SZ}=$ Tied mostly $\mathrm{Z} ; \mathrm{Z}=$ Tied all $\mathrm{Z}$.

Consistent tiers produced Half Knots and Figure Eights that were all or nearly all $\mathrm{S}$ or $\mathrm{Z}$.

Contrary tiers produced Half Knots that were all or nearly all opposite to their Figure Eights.

Mixed tiers produced equal numbers of $\mathrm{S}$ and $\mathrm{Z}$ enantiomers when tying Half Knots or Figure Eights or both.

Table 6. A comparison of Figure Eight Knots and cardinal knots according to number of tiers. Top: raw data. Bottom: similar cohorts combined.

\begin{tabular}{|c|c|c|c|c|c|c|}
\hline \multirow{2}{*}{$\begin{array}{l}\text { Cardinal Knots tied by } \\
\text { Figure Eight tiers }\end{array}$} & \multicolumn{5}{|c|}{ Number of Figure Eight tiers according to chirality } & \multirow{2}{*}{ Totals } \\
\hline & $S$ & $\mathrm{Sz}$ & Sz & sZ & Z & \\
\hline$s / S$ & 29 & 6 & 4 & 2 & 18 & 59 \\
\hline $\mathrm{Z} / \mathrm{Z}$ & 10 & 2 & 2 & 1 & 12 & 27 \\
\hline$S / Z$ & 3 & 1 & 2 & 1 & 6 & 13 \\
\hline $\mathrm{z} / \mathrm{S}$ & 3 & 0 & 1 & 0 & 8 & 12 \\
\hline Mixed & 16 & 3 & 1 & 3 & 4 & 27 \\
\hline Totals & 61 & 12 & 10 & 7 & 48 & 138 \\
\hline
\end{tabular}

\begin{tabular}{ccccc}
\hline Cardinal Knots tied by Figure Eight tiers & \multicolumn{3}{c}{ Number of Figure Eight tiers according to chirality } & \multirow{2}{*}{ Totals } \\
\cline { 2 - 4 } & S, SZ & SZ & SZ, Z & 59 \\
S/S & 35 & 4 & 20 & 27 \\
Z/Z & 12 & 2 & 13 & 13 \\
S/Z & 4 & 2 & 7 & 12 \\
Z/S & 3 & 1 & 7 & 27 \\
Mixed & 19 & 1 & 55 & 138 \\
Totals & 73 & 10 & & 7 \\
\hline
\end{tabular}

$\mathrm{S}=$ Tied all $\mathrm{S} ; \mathrm{SZ}=$ Tied mostly $\mathrm{S} ; \mathrm{sZ}=$ Tied $\mathrm{S}$ and $\mathrm{Z}$ equally; $\mathrm{SZ}=$ Tied mostly $\mathrm{Z} ; \mathrm{Z}=$ Tied all Z

The four Cardinal Knots are S/S and Z/Z Granny Knots, and S/Z and Z/S Reef Knots, where the chirality of the first Half Knot tied slash. 


\section{A COMPARISON OF ALL THREE TASKS}

Although there are similarities among the three tasks and a significant, and somewhat similar pattern of Figure Eight chiralities emerged in each case, the Overhand Knot task seems to be most analogous to the Figure Eight task and therefore yielded a higher percentage of consistent tiers. As summarized in Tables 7 and 8, the percentage of consistent or nearly consistent Figure Eight tiers is highest with the Overhand Knot data, while the percentages are lower for the other two basic tasks. Further, tiers who preferred the $S$ enantiomorph during basic tasks were less likely to tie contrary Figures Eight Knots than those who prefer to tie basic $Z$ enantiomorphs, and this agrees with data patterns observed in previous research [3]. $S$ tiers are more consistent than Z tiers.

The association among all four tasks may be more complex, and the data presented show a relationship in task chiralities. Nevertheless, the pattern is not straightforward, the data hint at underlying complexities, and more research is needed in order to reveal the mechanisms by which fewer tiers are consistently contrary or mixed in all tasks.

\section{DISCUSSION}

The data acquired demonstrate a link or relationship between Overhand Knot and Figure Eight Knot tying in terms of resulting chiralities. That relationship is less pronounced with Half Hitches and Half Knots in particular. The actions required to produce these knots are similar. However, mixed and contrary tiers demonstrated non-binary chirality patterns. Previous investigations into handedness and basic tying tasks - like Half Hitch, Half Knots, parcelling knots and shoelace tying - revealed similarly complex ratios [1-4]. Potential behavioural intricacies were suggested by the data accumulated from mixed and contrary tiers. This is reminiscent of patterns revealed in previous research concerning the complex correlation between shoelaces and parcel or binding knots, both of which require two wends rather than one wend $[1,3,4]$. Clearly, establishing an individual's tying habits or knot profile [1] entails the acquisition of multiple control samples from a variety of designated tasks.

Unfortunately, case evidence and even suspect samples are usually an incomplete snapshot of a more intricate set of behaviours and potential outcomes. Nevertheless, the fact that most survey respondents tended to produce Overhands and Figure Eights of equivalent chirality is a useful finding. First, the results verify that tying behaviour tends to be repeatable and that a favoured chirality can extend to other tying tasks. The presence of consistent Overhand Knots and Figure Eights in case evidence can demonstrate tying consistency, and comparisons to similar suspect samples can be corroborative in terms of group-characteristic behaviour.

However, associations must be made prudently. Context, the occurrence of accidental knots, the possibility of capsizement, and chirality reversal factors should be taken into account $[1-4,6]$. The analyser of evidence must understand what purpose any given knot served, and whether or not it was created accidentally. It is usually obvious when a knot is a residual structure, if the cord or rope had been used previously for other purposes. More germane to certain case analyses, scene details may indicate that a victim was bound by an assailant. In contrast, the feasibility of self-tying must be evaluated when the case is possibly or ostensibly a suicide or an autoerotic fatality. The reason is, overhead and self-tying procedures can be chiral reversal factors [1-3]. Other causes of chirality switching might include awkward ligature materials and unusual tying positions [4-8]. Furthermore,

Table 7. Proportion of Figure Eight tiers that were consistent, contrary or mixed, according to the three basic tying tasks.

\begin{tabular}{|c|c|c|c|c|}
\hline \multirow[t]{2}{*}{ Tasks } & \multicolumn{3}{|c|}{ Proportion of Figure Eight tiers for each tasks } & \multirow[t]{2}{*}{ Totals } \\
\hline & Consistent & Contrary & Mixed & \\
\hline \multirow[t]{2}{*}{ Overhand Knots } & 105 & 61 & 18 & 184 \\
\hline & $(57 \%)$ & $(33 \%)$ & $(10 \%)$ & \\
\hline \multirow[t]{2}{*}{ Half Hitches } & 56 & 35 & 34 & 125 \\
\hline & $(45 \%)$ & $(28 \%)$ & (27\%) & \\
\hline Half Knots & $\begin{array}{c}58 \\
(42 \%)\end{array}$ & $\begin{array}{c}45 \\
(33 \%)\end{array}$ & $\begin{array}{c}35 \\
(25 \%)\end{array}$ & 138 \\
\hline
\end{tabular}

Table 8. Percentage of consistent and nearly consistent Figure Eight tiers relative to contrary and nearly contrary Figure Eight tiers, according to the enantiomers in the three tying tasks.

\begin{tabular}{|c|c|c|c|c|}
\hline $\begin{array}{c}\text { Tying task } \\
\text { (Percentage of total, without mixed } \\
\text { tiers) }\end{array}$ & Task enanatiomers & $\begin{array}{c}\text { Number of consistent Figure Eight } \\
\text { tiers * }\end{array}$ & $\begin{array}{l}\text { Number of contrary } \\
\text { Figure Eight tiers * }\end{array}$ & $\begin{array}{l}\text { Percentage of contrary tiers relative to } \\
\text { consistent tiers }\end{array}$ \\
\hline Overhand Knot & $\mathrm{S}, \mathrm{Sz}$ & $67(S, S z)$ & $36(Z, s Z)$ & $54 \%$ \\
\hline (166 out of $184,90 \%$ ) & $z, s z$ & $38(Z, s Z)$ & $25(\mathrm{~S}, \mathrm{Sz})$ & $66 \%$ \\
\hline Half Hitch & $\mathrm{S}, \mathrm{Sz}$ & $31(\mathrm{~S}, \mathrm{Sz})$ & $16(Z, s Z)$ & $52 \%$ \\
\hline (91 out of $125,73 \%$ ) & $z, s z$ & $25(Z, s Z)$ & $19(\mathrm{~S}, \mathrm{Sz})$ & $76 \%$ \\
\hline \multirow{2}{*}{$\begin{array}{c}\text { Half Knot } \\
\text { (103 out of } 138,75 \%)\end{array}$} & $\mathrm{S}, \mathrm{Sz}$ & $43(\mathrm{~S}, \mathrm{Sz})$ & $24(\mathrm{Z}, \mathrm{sZ})$ & $56 \%$ \\
\hline & $\mathrm{Z}, \mathrm{sz}$ & $15(Z, s Z)$ & $21(\mathrm{~S}, \mathrm{Sz})$ & $140 \%$ \\
\hline
\end{tabular}

$S, Z=$ consistent tiers of these enetiomers

$\mathrm{Sz}, \mathrm{sZ}=$ nearly consistent tiers of $\mathrm{S}$ and $\mathrm{Z}$ enantiomers, respectively

Figure Eight enantiomers in brackets 


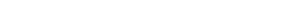

as mentioned at the outset, Bowlines can distort or capsize into Slip Knots (Figure 13), or more accurately Running Slip Loops, which share the basic Overhand structure. How a knot was tied and the potential for capsizement must be assessed before reliable comparisons can be made.

\section{LIMITATIONS}

The limitations of this study certainly suggest avenues of further inquiry. There were an insufficient number of left-handed respondents for comparisons with the dextral volunteers. There were more females in the sample than males. However, no apparent differences in tying patterns between gender groups were discerned in the data.

The biggest shortcoming was the lack of data concerning the Bowline. It requires a totally different set of manipulations, and a modified survey must be designed to acquire sufficient data and somehow compare the various tying methods to innate behaviour.

\section{CONCLUSIONS}

Based on these survey data, there is a higher likelihood that a tier will produce Figure Eight Knots which are equal in chirality to the Overhand Knots they habitually tie, since both tasks utilize similar manipulations of a single wend. This detail must be kept in mind when analysing case exhibits and suspect samples. However, the correlation is not perfect and a substantial percentage of respondents produced Overhand Knots and Figure Eight Knots of contrary or mixed chiralities. Concordance is apparent but less pronounced with Half Hitch and Half Knot tying tasks.

No significant general learning effect was detected in the data and the influence of chirality reversal factors - as examined in previous research was not investigated in this study. Nevertheless, the possibility of chirality reversal owing to situational interference cannot be ignored when assessing case evidence.

There were insufficient data to compare Bowlines to Overhand Knots. The two tasks are substantially different in execution and complexity, and there was an obvious learning effect in the small sample reported. This result was expected.

Clearly, more research is required. Other more sophisticated knots, which require similar tying actions and which also appear in case evidence, must be selected as suitable survey candidates for further study. These might include the Figure Nine or Intermediate Knot, sequences of Half Hitches and Overhand Knots, possibly the Sheet Bend, and loop versions of the Overhand, Figure Eight and Figure Nine Knots, to mention a few examples. Such an inquiry could focus also on basic and habitual tying actions such as turns, crossings, wraps and twists.

The data indicate that similar tying tasks may produce similar chiralities, and this observation can be applied to the examination of case evidence and the comparison of suspect samples, provided examiners of knot evidence understand that the correlation is not perfect and that situational factors may have confounded the tying process and caused chirality reversal. Chirality reversal factors must be clearly identified during the analysis, and they can include: overhead tying and the self-tying of neck ligatures, perhaps two-wend versus one-wend tying tasks, as well as awkward ligature materials and dimensions [2-8]. The likelihood of capsizements as well as residual and accident knots must be considered. With these caveats in mind, opportunities to link knot chiralities and similar tying actions must not be overlooked when examining ligature evidence.

\section{REFERENCES}

[1] Chisnall R., The forensic analysis of knots and ligatures, Lightning Powder Company, Inc., Salem, Oregon, 2000

[2] Chisnall R., What knots can reveal: the strengths and limitations of forensic knot analysis, J. Forensic Ident., 2007, 57, 726-749

[3] Chisnall R., Knot-tying habits, tier handedness and experience, J. Forensic Sci., 2010, 1232-1244

[4] Chisnall R., Tying anomalies and their significance in analysing knot evidence, Can. Soc. of Forensic Sc. J., 2009, 42, 172-194

[5] Chisnall R., Basic principles of forensic knot analysis: a qualitative study of tying behaviour, Investigative Sci. J., 2010, 2, 33-44, http://investigaivesciencesjournal. org/article/view/6687

[6] Chisnall R., An analysis of more than 100 cases involving knots and ligatures: knot frequencies, consistent tying habits and noteworthy outliers, Australian J. Forensic Sci., 2011, 43, 245-262

[7] Chisnall R., Structural recognition and nomenclature standardization in forensic knot analysis, Sci, Justice, 2016, 56, 282-301

[8] Budworth G., Identification of knots, J. Forensic Sci. Soc., 1982, 22, 327-331

[9] Nute H.D., Mirror images in knots, J. Forensic Sci., 1986, 31, 272-279

[10] Budworth G., Knots and crime, Police Review Publishing Co. Ltd., Great Britain, 1985

[11] Asher, H., The alternative knot book, Sheridan House, Great Britain, 1989

[12] Ashley C.W., The Ashley book of knots, Doubleday \& Company, Garden City, New York, 1944
[13] Berthier M.P.G., The art of knots, Macdonald and Jane's, London, Great Britain, 1978

[14] Bigon M., Regazzoni G., The Morrow guide to knots. Quill, William Morrow, New York, 1981

[15] Blandford P.W., Knots \& splices, Arco Publishing Company Inc., New York, 1965.

[16] Bourgault D., Guide pratique des noeuds, Éditions Coop Montpetit, Longueuil, Quebec, 1985, (In French)

[17] Brown T., Hunter R., Concise book of knots, Gage Publishing, Agincourt, 1977

[18] Budworth G., The knot book, Elliot Right Way Books, United Kingdom, 1983

[19] Budworth G., The ultimate encyclopedia of knots \& ropework, Lorenz Books, London, England, 1998

[20] Burgess, J.T., knots, ties \& splices, Coles Publishing Company Limited, Toronto, 1979

[21] Day C.L., Knots and splices, Granada Publishing, Great Britain, 1953

[22] Fletcher H., Knots ... and how to tie them, Coles Publishing Company Limited, Toronto, 1976

[23] Fry E.C., The Shell book of knots and ropework, Douglas, David \& Charles, Vancouver, 1977

[24] Gibson C., knots \& splices, Granada Publishing Limited, USA/Canada, 1979

[25] Graumont R., Hensel J., Encyclopedia of knots and fancy rope work, $4^{\text {th }}$ ed., Cornell Maritime Press, Inc. Cambridge, 1952

[26] Jacobson C., Knots for the outdoors, Falcon Guides, Guilford, Connecticut, U.S.A., 2008 
[27] Knots: a pocket companion, Strathearn Books Limited, Toronto, Canada, 1998

[28] Luebben C., Knots for climbers, Chockstone Press, Evergreen, Colorado, 1993

[29] Pawson D., Pocket guide to knots \& splices, Chartwell Books, Edison, New Jersey, 2001

[30] Raleigh D., Knots \& ropes for climbers, Stackpole Books, Mechanicsburg, Pennsylvania, 1998

[31] Shaw G.R., Knots: useful \& ornamental, Published by Collier Books, New York, 1933

[32] Warner C., A fresh approach to knotting and ropework, Charles Warner, Yanderra, New South Wales, 1992

[33] Wheelock W., Ropes, knots and slings for climbers, La Siesta Press, Glendale, California, 1960

[34] Microys H.F., Climbing ropes, 1977, Am. Alpine J., 130-147

[35] Warner C., Studies on the behaviour of knots, In: Turner J.C., van de Griend P. (Eds.), History and science of knots, Series on knots and everything, Volume 11, World Scientific, 1996

[36] Chisnall R., The O.R.C.A. rock climbing safety manual, $2^{\text {nd }}$ ed., Ontario Ministry of Tourism and Recreation, Ontario, Canada, 1985

[37] Annett M., The binomial distribution of right, mixed and left handers, Q. J. Exp. Psychol., 1967, 19, 327-334
[38] Annett M.A., Classification of hand preferences by association analysis, Br. J. of Psych., 1970, 61, 303-321

[39] Annett J., Annett M., Hudson P.T.W., Turner A., The control of movements in the preferred and non-preferred hands, Quart. J. Exper. Psychol., 1979, 31, 641-652

[41] Beaton A., Left side, right side, Yale University Press, New Haven, 1986

[40] Annett M., Five tests of hand skill, Cortex, 1992, 28, 583-600

[42] Dragovic M., Hammond G., A classification of handedness using the Annett hand preference questionnaire, Br. J. Psychol., 2007, 98, 375-387

[43] Todor J.I., Doane T., Handedness classification: preference versus proficiency, Percept. Mot. Skills, 1977, 45, 1041-1042

[44] Corey D.M., Hurley M.M., Foundas A.L., Right and left handedness defined: a multivariate approach using hand preference and hand performance measures, Neuropsy. Neuropsy. Be., 2001, 14, 144-152

[45] Chisnall R., The security of Bowlines and Figure Eight Loops as harness tie-ins: the principles of pre-knotting, post-knotting and concatenation, part 1, Knotting Matters, 2006, 90, 14-20

[46] Chisnall R., The security of Bowlines and Figure Eight Loops as harness tie-ins: the principles of pre-knotting, post-knotting and concatenation, part 2, Knotting Matters, 2006, 91, 18-21 